\title{
UDK 528.92 \\ MÖGLICHKEITEN UND UTOPIEN GEODÄTISCHER INFORMATIONSSYSTEME AUS DER SICHT EINES PRAKTIKERS
}

\author{
Ernst F. Simon
}

\author{
Received 200406 20; accepted 20040928
}

\begin{abstract}
The author comes to the conclusion that the variety of terms and the differences in interpretation existing in geodetic information systems must be restricted, and that all those systems have to be classified as geodetic information systems which are based on uniform coordinates, fundamental importance being attached to the field of land cadasters, i e real estate property as the basic system.

Instancing the simultaneous existence of systems in the historical development from the land tax register to a multipurpose register and the necessity of uniformity in surveying, not having been recognised in time, also with regard to the development of automation, the importance of a uniform geodetic information system is outlined on the basis of the land cadastre in the Federal Republic of Germany.

Citing the building cadastre and the service line cadastre as examples, the author explains that it is impossible to carry out any necessary material developments in the absence of certain basic conditions. Realisation of geodetic information systems is subject to political motivation, a wide-spread basic structure, classical protection of property and practical multipurpose use, all of them offering possibilities of interrelation.
\end{abstract}

Keywords: geodetic information system, land cadastre, building cadastre.

\section{Einleitung}

Für uns Geodäten ist in der Tat das Zeitalter der Informationssysteme angebrochen. Ich muß allerdings auch feststellen, dass diese Tatsache für einen Praktiker erst einmal nur sehr schwer zugängliche Fakten schafft, die sich schon aus der Vielfalt der verwendeten Begriffe für Informationssysteme ergeben. Es wird über Grundstücksdatenbank, raumbezogenes Informationssystem, kommunales Informationssystem, bodenbezogenes Informationssystem, topographisches Informationssystem, Landinformationssystem und geodätisches Informationssystem - Thema meiner eigenen Ausführungen gesprochen.

Daraus lässt sich unschwer die ganze Komplexität der ThemensteIlung erkennen. Ich bin daher als erstes gezwungen, eine Eingrenzung vorzunehmen und eine Definition für den Begriff "geodätisches Informationssystem" zu versuchen.

Wenn es Aufgabe der Geodäsie u. a. ist, das Festpunktfeld innerhalb eines Landes oder eines Gebietes koordinatenmäßig zu bestimmen, so dass dieses als Ausgangsbasis für topographische Vermessungen, Katastervermessungen und alle anderen vermessungstechnische Arbeiten dienen kann, dann kann man alle die Informationssysteme als geodätische Informationssysteme bezeichnen, die geodätisch bestimmte Daten eines Landes, einer Region oder eines Gebietes, die Koordinate oder unmittelbar davon abzuleitende Daten, also Geodaten, als Grundlage haben [1,2].

Die höchste Entwicklungsstufe eines geodätischen Informationssystems ist sicher das Landinformationssystem.

Aufgrund der Vielzahl geodätischer Informationssysteme muß ich also eine Eingrenzung vornehmen, indem ich mich auf den Teil der Geodäsie beschränke, der auch als Vermessungswesen bezeichnet wird, und hier wieder auf den Teilaspekt des Liegenschaftskatasters als nicht nur mögliches, sondern notwendiges Basissystem aller weitergehenden geodätischen Informationssysteme bis zum Landinformationssystem.

Mit meinen Ausführungen möchte ich den Versuch unternehmen, meine ganz subjektiven Gedanken und Überlegungen über die Entwicklungstendenzen und die Entwicklungen im deutschen Vermessungswesen seit Beginn der Automation hinsichtlich der Zielsetzungen Koordinatenkataster, Leitungskataster, Grundstücksdatenbank, Geodaten - mit automatisiertem Grundbuch, Liegenschaftsbuch, Gebäudebuch und Liegenschaftskarte als Grundlage oder Teil eines Landinformationssystems, eben die Entwicklung geodätischer Informationssysteme in diesem Bereich zu äußern.

Mit der Verwirklichung neuer Zielsetzungen und Verfahren, die Umstellung und Ausweitung der vorhandenen geodätischen Systeme zu Informationssystemen, werden dem Praktiker neue Aufgaben gestellt, die er vor Ort, auf der untersten arbeitstechnischen Ebene erfüllen muß. Ihn trifft es in vollem Umfang, wenn eine Entscheidung der zuständigen höheren administrativen oder politischen Instanz nur schwer praktikabel ist.

Beginn und Entwicklung der automatischen Datenverarbeitung wurde und wird von uns Geodäten mit Faszination verfolgt, denn unsere Aufgaben waren ja schon immer mit dem Problem behaftet, große Datenmengen in Registern, durch Rechenverfahren oder zeichnerisch zu bearbeiten und für Informationen in analoger Form möglichst aktuell zur Verfügung zu halten.

Die Faszination der Automation kann aber auch dazu verführen, die Möglichkeiten sachlicher und finanzieller Verwirklichung zu verkennen.

Der weiteren Behandlung meines Themas möchte ich als einen Ausschnitt der gegebenen Probleme folgende Thesen zu Grunde legen:

Als Praktiker muß ich nach dem augenblicklichen Stand der Entwicklung geodätischer Informationssysteme noch auf vorläufig nicht absehbare Zeit neben den 
modernen Informationssystemen weiter im herkömmlichen Liegenschaftskataster arbeiten, das z.T. über 150 Jahre alt ist.

Bei der Entwicklung der Automation wurde die Notwendigkeit einer Vereinheitlichung des Vermessungswesens nicht frühzeitig genug erkannt und damit z. T. vertan.

Am Beispiel Leitungskataster, als ein geodätisches Informationssystem aller unterirdischen Leitungen, zeigt sich, dass auch fachlich/sachlich gerechtfertigte Forderungen beim Fehlen der notwendigen Rahmenbedingungen nicht zu verwirklichen sind.

Bei der Verwirklichung der Grundstücksdatenbank sind insbesondere bei automatisiertem Gebäudebuch und bei der automatisierten Katasterkarte, d.h. in digitaler Form Tendenzen gegeben, die nicht die erwünschten und notwendigen Ergebnisse bringen.

Grundlage für ein umfassendes Landinformationssystem sollte bei den gegebenen Rahmenbedingungen nur ein geodätisches Informationssystem Liegenschaftskataster sein, also eine umfassende Geodatenbank, dessen Verwirklichung als Chance und Herausforderung für unseren Berufsstand nicht vertan werden darf.

\section{Zu meiner These Nr. 1}

Da ich überzeugt bin, dass ich bei dem augenblicklichen Stand der Informationssystementwicklung als Praktiker noch auf nicht absehbare Zeit in und mit dem herkömmlichen Liegenschaftskataster arbeiten muß, möchte ich über dessen Entstehen, Entwicklung und seinen heutigen Zustand, aus dem für die zukünftigen Entwicklungsnotwendigkeiten Analogschlüsse gezogen werden sollten, einen kurzen Abriss geben.

Ende des 18. und im 19. Jahrhundert wurde in allen Deutschen Staaten das Grundsteuerkataster aufgestellt und eine Landesvermessung durchgeführt. Diese Aufgaben und ihre Durchführung waren ganz klar und eindeutig politisch begründet und motiviert.

Mit der Einführung des Grundbuchs - 1872/1900 wurde das Grundsteuerkataster zum Liegenschaftskataster, d. h. zur vermessungstechnischen Grundlage für den Nachweis des Eigentums am Grund und Boden. Die Bezeichnung des Liegenschaftskatasters als Mehrzweckkataster wurde, soviel mir bekannt ist, erstmalig durch die Formulierung der Aufgabenzuweisungen im Katastergesetz des Volksstaates Hessen 1926 initiiert. Mit Liegenschaftskataster und Grundbuch war also ein geodätisch/rechtlicher Nachweis geschaffen worden, der seine beiden ursprünglichen Aufgaben, umfassende Information über das Eigentum und allen damit verbundenen Rechten und Belastungen und die Daten der Besteuerung des Grund und Bodens zu liefern, erfüllte. Das Grundbuch, basierend auf einem Reichsgesetz, kann man als bundeseinheitlich bezeichnen. Das Liegenschaftskataster entstand aber länderspezifisch, deren Gebiete sich mit den heutigen Bundesländern nur ausnahmsweise decken. So gibt es in den einzelnen Bundesländern in ihrer technischen Ausführung und Qualität noch sehr unterschiedliche Liegenschaftskataster. Auch in den neueren Vermessungs- und Katastergesetzen, die in fast allen Bundesländern nach 1945 entstanden, wurden die Aufgaben des Liegenschaftskatasters so formuliert allerdings politisch und rechtlich mit unterschiedlicher Intensität, wie sich heute erweist, dass man von Mehrzweckkataster sprechen kann.

Man kann zwar sagen, dass das Liegenschaftskataster bis heute in großen Bereichen Unterlagen und Informationen für Planungsaufgaben, für Bebauungspläne und Bauprojekte im Hoch- und Tiefbau liefern konnte. Man muß aber auch feststellen, dass es den umfassen den Anforderungen, die an ein Mehrzweckkataster zu stellen sind, niemals gerecht geworden ist.

Den Grund für diese Entwicklung sehe ich in ganz großen Zügen in folgenden zwei Faktoren.

Die Vermessungs- und Katasterverwaltungen setzten die Schwerpunkte und Prioritäten ihrer Aufgaben nicht richtig. Statt die in sehr viel größerem Umfange notwendige Erneuerung und Modernisierung des Liegenschaftskatasters zu betreiben, wurden in erster Linie Fortführungsvermessungen und Auftragsarbeiten für Dritte durchgeführt. Prof. Matthias (Zürich) hat das hier vorhandene Problem wie folgt formuliert: "Ein Individium oder ein System, das sowohl mit Führungsals auch mit Ausführungsaufgaben beauftragt ist, wird die Führung stark vernachlässigen und sich immer der Ausführung, der Emsigkeit, zuwenden" [3].

Unserem Berufsstand insgesamt, und hier insbesondere dessen höchsten Repräsentanten, ist es nicht oder nicht ausreichend gelungen, die Politiker und die politischen Instanzen von der volkswirtschaftlichen Bedeutung eines Mehrzweckkatasters zu überzeugen. Ein entsprechend großer "Nutzungshunger" bei Planungsinstitutionen, Wirtschaft und Staatsbürgern, der eine wirkungsvolle Resonanz bei den Politikern erzeugt hätte, konnte auch nicht geweckt werden. Wenn ich einmal von der fehlenden Einmessungspflicht für unterirdische Leitungen absehe, war der gesetzliche Rahmen dafür gegeben. Er wurde aber materiell nicht ausgefüllt.

\section{Nun komme ich zu meiner These Nr. 2}

Mit der Entwicklung der automatischen und elektronischen Datenverarbeitung konnten wir Geodäten ab Mitte der 50er Jahre größere vermessungstechnische Datenmengen rechnerisch und zeichnerisch, wirtschaftlich und schnell verarbeiten und bearbeiten. Es entstand ebenfalls die Möglichkeit, die in Form von Büchern und Karteien, handschriftlich oder mit Schreibmaschine geführt, vorhandenen Grundstücksinformationen in EDV geführte Datenbanken umzuarbeiten. Allerdings beschritt bei der numerischen Datenverarbeitung jedes Land, und in jedem Land auch noch jede Institution, eigene Wege. Ähnliches gilt für die Einführung von Datenverarbeitungsverfahren für das Liegenschaftsbuch, wo fünf verschiedene Verfahren im Einsatz waren, und ein sechstes Verfahren, genannt GAL (Gemeinschaft der Anwender des automatisierten Liegenschaftsbuches), für alle Länder eine Vereinheitlichung bringen sollte. 2003 kann man allerdings 
feststellen, dass diese Vereinheitlichung gescheitert ist. Die heute eingesetzten Verfahren sind je nach Bundesland sehr unterschiedlich, nach dem Motto, jede Verwaltung glaubt den „Stein der Weisen“ gefunden zu haben.

Die Entwicklung der Datenverarbeitung verlief in den einzelnen Ländern also sehr uneinheitlich und unterschiedlich und hat im vermessungstechnischen Bereich bei den vorhandenen Programmsystemen Fakten geschaffen, die ausschließlich aus Gründen der Vereinheitlichung wohl kaum wieder rückgängig gemacht werden können. Welche Auswirkungen dieses Faktum für die Verwirklichung eines umfassenden Landinformationssystems haben wird, ist sicher noch nicht voll abzusehen.

Dazu möchte ich weiter noch feststellen, dass die Automatisierung des Liegenschaftsbuches nach dem derzeitigen Stand für den praktischen Nutzer lediglich eine verwaltungsinterne Maßnahme bedeutet, die $\mathrm{z}$. Zt. jedenfalls nicht mit einem Zuwachs an Information verbunden ist, und die auch nicht nur Vorteile gebracht hat.

Hinzu kommt, dass es volkswirtschaftlich schon schwer verständlich ist, dass das neu erstellte automatisierte Liegenschaftsbuch der Vermessungsverwaltungen an die Justiz- und Finanzverwaltungen in Form von Papierauszügen tonnenweise abgegeben wird, anstatt entsprechende EDV - Speichermedien einzusetzen. Ich gehe aber davon aus, dass durch die Entwicklung in der Elektronik das Preis- Leistungsverhältnis so günstig wird, dass von daher eine Dezentralisierung, in der ich die Zukunftsentwicklung sehe, möglich wird. Nur unter diesen Bedingungen kann der Vorteil der Automation des Liegenschaftsbuchs voll genutzt werden. Für die Öffentlich bestellten Vermessungsingenieure als Vermessungsstellen im öffentlichen Vermessungswesen muß die Möglichkeit des direkten Zugriffs online, wie das in Österreich für unsere Kollegen selbstverständlich ist, gegeben sein. Diese Forderung, die ich schon im Jahre 1981 gestellt habe, ist im Jahr 2003 erst in einigen wenigen Bundesländern realisiert worden, z.B. in Hessen.

\section{Ich komme zu meiner These Nr. 3}

Vor wenigen Jahren schlugen die Diskussionen über ein Leitungskataster, über das Für und Wider, hohe, sogar sehr hohe Wellen. Seit geraumer Zeit ist es sehr ruhig darum geworden. Damit keine Missverständnisse entstehen, ich persönlich bin auch heute noch von der objektiven Notwendigkeit eines umfassenden und einheitlichen Leitungskatasters überzeugt.

Ich kann die Auffassung von Lutz (Dortmund) z. B. nicht teilen [4], dass bei der Planung oder im Rahmen eines Bauvorhabens eine so große Anzahl von Trägern öffentlicher Belange $\mathrm{zu}$ beteiligen sei, dass es möglicherweise auf die Träger von Strom, Gas, Wasser und Telefon auch nicht mehr ankomme. Ich halte diese Auffassung im Hinblick auf unsere gesamte wirtschaftliche Entwicklung sogar für sehr gefährlich, denn die Planungsprozesse dauern immer länger.
Ich kann zum Leitungskataster heute eigentlich nur feststellen, dass meiner Meinung nach die richtige Idee falsch vermarktet worden ist, wenn ich einmal so sagen darf.

Jedenfalls konnten weder die Politiker noch die Leitungsbetreiber, noch die Nutzer - hier vor allem Planungsinstitutionen und Baufirmen - von der Notwendigkeit und dem Nutzen eines Leitungskatasters überzeugt werden, obwohl nach verschiedenen Veröffentlichungen und auch auf Grund kleiner Pilotprojekte die Kosten-Nutzen-Untersuchungen positive Ergebnisse brachten.

Im gleichen Zeitraum ist von den Politikern in allen Bundesländern oft gegen den Widerstand der Beteiligten, eine Regionalreform mit exorbitanten Kostensteigerungen - allein die Personalkapazitäten wurden auf das Doppelte bis Dreifache erhöht - bei der kommunalen Selbstverwaltung verwirklicht worden. Ich wollte mit diesen kurzen Ausführungen zum Leitungskataster nur auf die immense Bedeutung politischer Bewusstseinsbildung hinweisen, die auch bei meiner These Nr. 4 zur Verwirklichung der Grundstücksdatenbank eine entscheidende Rolle spielen wird.

Anfang der 70er Jahre wurde aus den technischen Möglichkeiten der Automation, man muß sagen konsequenter Weise, die Idee der Grundstücksdatenbank entwickelt, wobei man, sachlich durchaus richtig, davon ausging, dass Grundbuch und Liegenschaftskataster in einer integrierten Grundstücksdatenbank vereinigt werden sollten. Die. volle Verwirklichung dieses Beschlusses wäre in der Tat zukunftsweisend gewesen. Aber heute muß man wohl realistischer Weise feststellen, dass die integrierte Grundstücksdatenbank nur in marginalen Ansätzen verwirklicht ist.

Wir haben also vorerst nur Teillösungen erhalten.

Für das automatisierte Gebäudebuch - der Nachweis der Gebäude und Bauwerke gehört m. E. unabdingbar in erster Priorität zu einem Landinformationssystem - ist zwar ein Konzept entwickelt. Es hat sich aber, so weit mir bekannt ist, bis heute kein Bundesland oder ein entsprechender Zusammenschluss bereit gefunden, eine Realisierung in Angriff zu nehmen. Wenn sich auch nach der Nutzen-Kosten-Untersuchung der Firma Diebold $\mathrm{GmbH}$ durch die automatisierte Liegenschaftskarte angeblich kaum Konsequenzen für den Außendienst ergeben, so erhalten wir in der Praxis zu den vorhandenen heterogenen Liegenschaftskatastern erst einmal eine weitere Heterogenität hinzu.

Erst mit der Fertigstellung der automatisierten Liegenschaftskarte - ALK -, d. h. einem geodätischen Informationssystem für den numerischen und graphischen Teil des Liegenschaftskatasters, so möchte ich es einmal bezeichnen, wird Homogenität und der volle Nutzen der Automation erreicht, wenn denn die Digitalisierung der notwendigen Genauigkeit durch eine Erneuerung entspricht, was aber in weiten Bereichen nicht der Fall ist, da man einfach die analogen Liegenschaftskarten graphisch digitalisiert hat.

Bei uns in Deutschland hat sich, meiner Meinung nach, eine unheilvolle Tendenz entwickelt, vor allem auf 
den Gebieten der Gesetzgebung, des Rechts und der Verwaltung, alles so perfekt wie möglich und für jeden auch nur theoretisch denkbar möglichen Fall zu regeln. Ich sehe diese Gefahr auch bei dem Auf- und Ausbau der geodätischen Informationssysteme. Als Geodät der Praxis und als Staatsbürger müssen mir erhebliche Bedenken kommen, wenn ich die doch sicher kompetenten Veröffentlichungen über die derzeitige Planung der automatisierten Liegenschaftskarte gelesen habe, und die realistische Verwirklichung sehe [5]. Als Praktiker habe ich tagtäglich mit den konsumptiven, vermessungstechnischen Bedürfnissen des Staatsbürgers - als Grundstückseigen-tümer, als Bauherr, mit Architekten, Bauträgern, Planerneben auch privaten - und mit der Verwaltung zu tun. Dabei muß ich leider immer wieder erkennen, dass es selbst für die Erfordernisse, z. B. der Gebäudeeinmessungspflicht für das bestehende Liegenschaftskataster, an Verständnis mangelt - und das nicht nur bei Privateigentümern.

Dem Staatsbürger und den Politikern die Finanzierbarkeit und den Nutzen der Umwandlung des Liegenschaftskatasters in ein geodätisches Informationssystem und durch digitale Erfassung aller Infrastrukturdaten $\mathrm{zu}$ einem Landinformationssystem $\mathrm{zu}$ erweitern und zu ergänzen plausibel darzulegen, dürfte $m$. E. eine höhere Präferenz haben als die Lösung der Detailprobleme [6, 7]. Für mich ist die Schaffung geodätischer Informationssysteme nicht in erster Linie ein Problem der technischen Möglichkeiten, sondern ein Problem der ökonomischen und gesellschaftlichen Philosophie. Und hier sehe ich eine große Gefahr.

Für jedes geodätische Informationssystem ist unbestritten unabdingbare Forderung, dass jede Information nur mit der notwendigen Genauigkeit erfasst und gespeichert werden soll. Da wir in Deutschland aber in überwiegendem Maße für die Eigentumsgrenzen einen Zahlennachweis, also ein numerisches Liegenschaftskataster haben, muß ein geodätisches Informationssystem die Informationen über die Eigentumsgrenzen mit entsprechender Genauigkeit nachweisen [8]. Ich halte es z. B. für ein Unding, dass die Informationen für die Eigentumsgrenzen und die Gebäude und Bauwerke in einem Informationssystem durch Digitalisierung der Katasterkarten erfasst werden, wenn gleichzeitig im Planungs- und Baurecht für Baulasten oder Grenzabstände cm-Genauigkeit verlangt wird.

An ein geodätisches Informationssystem ist daher die Forderung zu stellen, dass die Eigentumsgrenzen jederzeit in der Örtlichkeit mit vertretbarem Aufwand reproduzierbar sind, damit das Eigentum an Grund und Boden gewährleistet und gesichert werden kann. Mich als Praktiker interessiert daher ungemein, dass ich die für meine Arbeit notwendigen Einzelinformationen auf aktuellem Stande möglichst schnell erhalte, und dass das Ergebnis meiner Arbeit so schnell wie möglich in das Informationssystem eingegliedert wird.

Auch ohne Automationsfachmann zu sein, ist meine Feststellung sicher nicht falsch, dass die automatisierte Liegenschaftskarte mit der Einarbeitung jeder zusätzlichen Information und mit jeder Vergrößerung der Datenmenge in ihrer Komplexität. überproportional ansteigt, und dass es bei dieser Entwicklung Grenzen gibt, wo die notwendigen personellen Voraussetzungen bei dem gegebenen Personaldurchschnitt in der Praxis nicht mehr erfüllt werden können. Auf die Gefahr der Überinformation, auf die ebenfalls Prof. Zemanek in einem Festvortrag eingegangen ist, möchte ich nur hinweisen. Eine Automationseuphorie, die den Menschen vergisst, wird der notwendigen Realisierung nicht nutzen. Meiner Ansicht nach gilt es, hier die notwendigen Grenzen der Beschränkung zu erkennen, aber auch die notwendigen Rahmenbedingungen.

Ich meine, nach Jahren, in denen wir Praktiker uns automationsgerechten Verfahren anpassen mussten, muss die Elektronik dazu nutzbar gemacht werden, praxisgerechte Automationsverfahren zu entwickeln, die auch eine entsprechende Optimierung in der Bearbeitung gewährleisten.

Damit komme ich zu meiner 5. und letzten These, dass die Grundlage für ein Landinformationssystem nur ein geodätisches Informationssystem Liegenschaftskataster sein sollte, dessen Verwirklichung eine Chance und eine Herausforderung an uns Geodäten ist, die wir richtig nutzen müssen.

Das Liegenschaftskataster ist das einzige flächendeckende geodätische System, auf dessen Grundlage ein Landinformationssystem entwickelt werden kann, mit allen Vorteilen, dass wir es grundsätzlich schon haben, und allen Nachteilen, dass es historisch bedingt sehr uneinheitlich, unterschiedlich in der Qualität und noch sehr unvollständig für die Anforderungen an ein geodätisches Informationssystem ist, und dass die historischen Entwicklungen aus rein rechtlichen Gründen auch in einem modernen Informationssystem erfasst werden müssen. Dazu ist festzustellen, dass das Liegenschaftskataster eigentlich die besten Grundlagen für die Erstellung eines geodätischen Informationssystems dort bietet, wo die Informationen eines LIS (Landinformationssystem), oder auch Geodatenbank, für einen großen Teilbereich in Zukunft nur noch wenig gebraucht werden, nämlich in Gebieten, in denen Planung und Bebauung abgeschlossen sind, z. B. in Trabantenstädten, Baugebieten, in flurbereinigten Gemarkungen, bei denen im Zuge der Neuordnung des Ländlichen Raumes auch Neubaugebiete bearbeitet wurden usw.

Ich meine also, dass mit dem Aufbau eines geodätischen Informationssystems Liegenschaftskataster dort begonnen werden muß, wo in absehbarer Zeit auf dem Gebiet der Planung, Sanierung, Bebauung usw. etwas geschehen wird. Erst dann können wir als Geodäten doch den vollen Nutzen eines solchen Systems den anderen Disziplinen beweisen. Das erfordert allerdings ein vollständiges Umdenken bei den Katasterverwaltungen über Prioritäten und auch Verfahren der Katastererneuerung - auf das Problem der Aufgabenverteilung zwischen Verwaltung und freiem Beruf möchte ich nur hinweisen - und erfordert, sozusagen Marktforschung zu betreiben, wo Katastererneuerung für zukünftige Entwicklungen notwendig werden. Den Aufbau eines Landinformationssystems über das geodätische 
Informationssystem Liegenschaftskataster nur dort, wo Koordinaten schon vorhanden sind und dann den Ausbau über allmähliche oder partielle Erneuerung, halte ich für den falschen Weg, der uns letztlich nicht $\mathrm{zu}$ dem gewünschten Ziel führt.

Aus dem Liegenschaftskataster ein geodätisches Informationssystem $\mathrm{zu}$ einem Landinformationssystem $\mathrm{zu}$ entwickeln, ist eine riesige Aufgabe. In einer Projektstudie nur für den Kreis Recklinghausen 1980/81 heißt es z. B. "Zum Aufbau einer Koordinatendatenbank sind bei z. Zt. ca. 220000 Flurstücken 5 Millionen Vermessungspunkte $\mathrm{zu}$ bestimmen und $\mathrm{zu}$ speichern. Für ein einheitliches Katasterkartenwerk sind noch 750 Rahmenkarten 1: 1000 herzustellen und 545 Rahmenkarten zu ergänzen. Bei bisherigen Arbeitsverfahren eine Aufgabe von 50 bis 60 Jahren. Nur mit Einsatz der graphischen Datenverarbeitung kann dieser Zeitraum erheblich verkürzt werden" [9].

Um nur annähernd einen Eindruck von dem Umfang der Aufgaben für ein Landinformationssystem zu vermitteln, möchte ich einige statistische Zahlen für Deutschland für das Jahr 1999/2000 nennen.

Aus dem neusten mir zur Verfügung stehenden Statistischen Jahrbuch 2001 für die Bundesrepublik Deutschland [10, 11] ergeben sich folgende für ein Landinformationssystem auf der Basis geodätischer Daten digital zu erfassender Infrastrukturdaten von wesentlicher

Bedeutung. Wir haben

1. 13850 Gemeinden - von unter 100 bis über 500.00 Einwohner -

2. ca. 41.409 Gemarkungen

3. ca. 62.177.800 Flurstücke

4. ca. 1,35 Milliarden Vermessungspunkte

5. 16.583.053 Wohnhäuser (1999)

6. 37.984.000 Wohnungen (1999)

7. $230.735 \mathrm{~km}$ Straße des überörtlichen Verkehrs, davon

7.1. $11.515 \mathrm{~km}$ Autobahnen

7.2. $41.321 \mathrm{~km}$ Bundesstraßen - mit $9.830 \mathrm{~km}$ Ortsdurchfahrten

7.3. $86.823 \mathrm{~km}$ Landesstraßen - mit $19.746 \mathrm{~km}$ Ortsdurchfahrten

7.4. $91.076 \mathrm{~km}$ Kreisstraßen - mit $19.656 \mathrm{~km}$ Ortsdurchfahrten

8. ca. $420.000 \mathrm{~km}$ Gemeindestraßen

9. $\quad 80.447 \mathrm{~km}$ Eisenbahnstrecken - davon $46.373 \mathrm{~km}$ elektrifiziert

10. über 2,7 Milliarden $\mathrm{km}$ unterirdischer Versorgungsleitungen (Abwasserkanäle, Gasversorgungsleitungen, elektrische Versorgungskabel, Telefonkabel)

11. ca. $700.000 \mathrm{~km}$ oberirdische Versorgungsleitungen (vor allem elektrische Hochspannungsleitungen)

12. 37 Seen mit einer Fläche von $1370,3 \mathrm{~km}^{2}$

13. 30 Talsperren mit einer Maximalfläche von $161,2 \mathrm{~km}^{2}$

14. 15 Schifffahrtskanäle mit einer Gesamtlänge von $1.555,6 \mathrm{~km}$

15. 41 Flüsse mit einer schiffbaren Länge von $5.662 \mathrm{~km}$ 16. $\quad 7.265 \mathrm{~km}^{2}$ Nationalparks
17. $16.134 \mathrm{~km}^{2}$ Biosphärenreservate

18. $\quad 8.704 \mathrm{~km}^{2}$ Naturschutzgebiete

19. $\quad 67.588 \mathrm{~km}^{2}$ Naturparke

20. 17 Großflugplätze mit 9000 bis 228000 gestarteten Flugzeugen/Jahr - daneben gibt es noch eine ungenannte Zahl von Flug- und Landeplätzen mit insgesamt 3.430.000 gestarteten Flugzeugen (Motor- und Segelflugzeuge) -

21. 471.980 Landwirtschaftliche Betriebe (1999)

22. 2.886.268 Wirtschaftsbetriebe insgesamt (2000) Deutschland hat eine Gesamtgröße von $357.022 \mathrm{~km}^{2}$. Davon sind.

Gebaute Flächen 26.716 km² $^{2}$ 7,483\% der Fläche Landwirtschaftliche Flächen $216.610 \mathrm{~km}^{2}(60,671 \%$ der Fläche)

Wälder und naturnahe Flächen $108.210 \mathrm{~km}^{2}$ (30,309\% der Fläche) Wasserflächen $3.798 \mathrm{~km}^{2}$ (1,064\% der Fläche) Feuchtfläche $1.689 \mathrm{~km}^{2}(0,473 \%$ der Fläche $)$

An der Größenordnung dieser Infrastrukturdaten, die absolut keinen Anspruch auf Vollständigkeit erheben, kann man aber ersehen, welche riesige Aufgabe die Schaffung eines Landinformationssystems bedeutet.

Es sind Kostenermittlungen und Kostenschätzungen für diese riesige Aufgabe versucht worden, denen ich aus Gründen der Unsicherheit, die im Augenblick alle diese Aussagen haben, keine weitere hinzufugen möchte.

\section{Schlussfolgerungen}

Als Resümee meiner Überlegungen $\mathrm{zu}$ den Möglichkeiten des Aufbaus eines geodätischen Informationssystems Liegenschaftskataster als Grundlage für ein Landinformationssystem, die ich wegen des Umfanges nur skizzenhaft darstellen kann, möchte ich folgende Schluss- folgerungen ziehen.

Die Entwicklung eines geodätischen Informationssystems bringt für den Praktiker während der Entstehungsphase sicher keine Entlastung, sondern eher zusätzliche Belastung, die er im Interesse des zukünftigen Nutzens aber akzeptieren muß.

Auch für ein geodätisches Informationssystem gilt die Maxime, die Benzenberg schon für die Aufstellung des Katasters nannte. Das wichtigste ist, dass es fertig wird. Eine EDV- Generationsphase beträgt inzwischen 2 bis 3 Jahre, oder noch kürzer.

Für die Realisierung der von uns als richtig und notwendig erachteten Aufgabe genügt nicht deren Postulierung, sondern wir brauchen die Überzeugung der Politiker und politische Motivierung als Unterstützung. Dazu ist es aber notwendig, dass nicht nur die Interessen der Verwaltungen artikuliert werden, sondern der Nutzen für die Wirtschaft und den Staatsbürger, die ja letzten 
Endes auch die Kosten aufbringen müssen. Das A und O für die Schaffung eines Landinformationssystems auf der Grundlage von Daten eines geodätischen Informationssystems des Liegenschaftskatasters, ist der politische Wille und die notwendigen Geldmittel. Die technische Ausführung werden wir Geodäten schon schaffen.

Bei der Realisierung sollte nur das unbedingt Notwendige mit der notwendigen Genauigkeit und nicht höchste Perfektion verwirklicht werden. Man sollte bei der Realisationsphase auch mehr Geodäten aus der Praxis und deren praxisbezogene Bedürfnisse berücksichtigen. Die Praktiker müssen nachher schließlich mit dem System arbeiten.

Bei der zu erkennenden Entwicklung der elektronischen Datenverarbeitung bin ich der Meinung, das sowohl aus sachlichen als auch aus organisatorischen Gründen der Dezentralisierung gegenüber der Zentralisierung der Vorzug gegeben werden muss. Allein die Bindung an ausschließlich Großrechnersysteme halte ich für gefährlich.

Die beteiligten Verwaltungen müssen im Interesse einer einheitlichen Verfahrens- und Programmentwicklung, so weit dies noch möglich ist, kompromissbereit hinsichtlich ihrer Kompetenzen und Sonderinteressen sein.

Die Entwicklung eines geodätischen Informationssystems Liegenschaftskataster erfordert auch Umdenkungsprozesse in der Verwaltung. Es müssen andere Prioritäten als bisher gesetzt werden. Wenn das öffentliche Vermessungswesen in der Lage ist, die Bedürfnisse der Nutzer seines Informationssystems voll und ganz zufrieden $\mathrm{zu}$ stellen, ergeben sich automatisch die ManagementFunktionen für die weitergehenden oder anderen geodätischen Informationssysteme. Es wird so lange jeder potentielle Nutzer seine eigenen Vermessungen durchführen und die benötigten Pläne und Karten ergänzen bzw. selbst herstellen, wie das Vermessungswesen nicht in der Lage ist, die entsprechenden Bedürfnisse zu erfüllen.

Es geht alles in allem für uns Geodäten darum, die Faszination der Automation in realistische Bahnen $\mathrm{zu}$ lenken und innerhalb der möglichen Grenzen zu verwirklichen.

\section{Literatur}

1. Eichhorn, G. Zielsetzung und Organisation von Landinformationssystemen. BDVI-Forum 1979/80, S. 329.

2. Herzfeld, G. Vorstufen zur Grundstücksdatenbank Unter besonderer Berücksichtigung der Verhältnisse in Hessen und Rheinland-Pfalz. Vortrag auf der fachwissenschaftlichen Tagung des DVW Hessen am 10.5. 1982 in Limburg.

3. Matthias, H. Staat oder freier Beruf als Unternehmer. Schweiz. Zeitschrift für Vermessungswesen, Photogrammetrie u. Kulturtechnik, Nr. 2/1975, Synthese zu diesem Aufsatz 1982 u. V.

4. Lutz, W. Ist der Nachweis von Versorgungsleitungen eine hoheitliche Aufgabe der öffentlichen Verwaltung oder eine privatwirtschaftliche der Leitungsträger? ZfV 102, 1977, S. 414-417.

5. Mittelstraß, G. Die Automatisierte Liegenschaftskarte, Stand nach 2,5 Jahren Forschungs- und Entwicklungsarbeit. ZfV 104, 1979, S. 554-566.

6. Rose, W. Kosten und Nutzen eines Leitungskatasters. ZfV 103, 1978, S. 554-569.

7. Schlehuber, J. Die Grundstücksdatenbank. ZfV 1977. 102, S. 532-540.

8. Schuster, O. Gedanken zu einem Landinformationssystem in Deutschland. BDVI-Forum 1979/1980, S. 339.

9. Simon, E. F. Politische und wirtschaftliche Probleme des öffentlichen Vermessungswesens - Chancen des freien Berufs? BDVI-Forum 1979/80, S. 123. Projektstudie Teil I. Elektronische Datenverarbeitung im Kataster- und Vermessungswesen, Kreis Recklinghausen 1980/81.

10. Statistisches Bundesamt: Statistisches Jahrbuch 2001 für die Bundesrepublik Deutschland.

11. Recherchen: ABV (Arbeitsgemeinschaft der Vermessungsverwaltungen der Länder der Bundesrepublik Deutschland) und Hessisches Landesvermessungsamt Wiesbaden, 2003.

\section{GEODEZINIŲ INFORMACINIŲ SISTEMŲ GALIMYBĖS PRAKTIKŲ POŽIŪRIU}

\section{Ernst F. Simon}

S antrauka

Daroma išvada, kad turi būti apribota geodezinių sistemų terminu ivairove ir skirtingas interpretavimas. Visos tokios sistemos turi būti suklasifikuotos pagal bendras koordinačiu sistemas, daugiausia dėmesio kreipiant i jų reikšmę žemės kadastrui. Pavyzdžiui, Vokietijos Federacinèje Respublikoje plečiant žemès taksacijos registrą iki daugiafunkcinio žemès registro tuo pačiu metu buvo taikomos ir kitos geodezinès sistemos. Taigi laiku nebuvo atkreiptas dèmesys i geodeziniu matavimu vientisumą ir bendrumą bei būtinybę tobulinti automatizuotas matavimo sistemas. Pateikiama pavyzdžių iš miestų statinių kadastro ir sklypų ribų kadastro, kuriais įrodoma, kad neįmanoma atlikti miestų plètros uždavinių nesant apibrežtų pagrindinių sąlygų. Taigi geodezinių informaciniu sistemu plètra tampa politinès motyvacijos objektu, plačia pagrindine ir iš esmès daugiatiksle struktūra, klasikiniu nuosavybès apsaugos garantu.

Raktažodžiai: geodezinès informacinès sistemos, žemès kadastras, pastatų kadastras. 